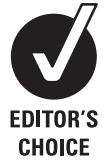

${ }^{1}$ Department of

Gastroenterology, University Hospital Gasthuisberg, Leuven, Belgium; ${ }^{2}$ Department of Paediatric Gastroenterology, University Hospital Gasthuisberg, Leuven, Belgium

${ }^{3}$ Department of Electrical Engineering and Computer Science-Montefiore Institute University of Liege, Belgium

Correspondence to: Professor P Rutgeerts, Department of Gastroenterology, University Hospital Gasthuisberg, Herestraat 49, B-3000 Leuven, Belgium paul.rutgeerts@uz.kuleuven.be

Revised 14 July 2008 Accepted 5 August 2008 Published Online First 2 October 2008

\title{
Long-term outcome of treatment with infliximab in 614 patients with Crohn's disease: results from a single-centre cohort
}

\author{
F Schnitzler, ${ }^{1}$ H Fidder, ${ }^{1}$ M Ferrante, ${ }^{1}$ M Noman, ${ }^{1}$ I Arijs, ${ }^{1}$ G Van Assche, ${ }^{1}$ I Hoffman, ${ }^{2}$ \\ K Van Steen, ${ }^{3}$ S Vermeire, ${ }^{1}$ P Rutgeerts ${ }^{1}$
}

\begin{abstract}
Background and aims: This observational study assessed the long-term clinical benefit of infliximab (IFX) in 614 consecutive patients with Crohn's disease (CD) from a single centre during a median follow-up of 55 months (interquartile range (IOR) 27-83).
\end{abstract}

Methods: The primary analysis looked at the proportion of patients with initial response to IFX who had sustained clinical benefit at the end of follow-up. The long-term effects of IFX on the course of CD as reflected by the rate of surgery and hospitalisations and need for corticosteroids were also analysed.

Results: $10.9 \%$ of patients were primary non-responders to IFX. Sustained benefit was observed in 347 of the 547 patients (63.4\%) receiving long-term treatment. In 68.3\% of these, treatment with IFX was ongoing and in $31.7 \%$ IFX was stopped, with the patient being in remission. Seventy patients $(12.8 \%)$ had to stop IFX due to side effects and $118(21.6 \%)$ due to loss of response. Although the yearly drop-out rates of IFX in patients with episodic $(10.7 \%)$ and scheduled treatment $(7.1 \%)$ were similar, the need for hospitalisations and surgery decreased less in the episodic than in the scheduled group. Steroid discontinuation also occurred in a higher proportion of patients in the scheduled group than in the episodic group.

Conclusions: In this large real-life cohort of patients with $C D$, long-term treatment with IFX was very efficacious to maintain improvement during a median follow-up of almost 5 years and changed disease outcome by decreasing the rate of hospitalisations and surgery.

Crohn's disease (CD) is a disabling chronic relapsing inflammatory disorder of the bowel. Long-term experience with standard therapies for inflammatory bowel disease (IBD) up to the late 1990s demonstrated diverse limitations of these therapies in the treatment of IBD. Several population-based studies reported on the inability of corticosteroids to maintain remission, although these drugs have good short-term efficacy. ${ }^{1-5}$ Immunomodulators (IMMs) including azathioprine (AZA), its metabolite 6mercaptopurine (6-MP) and the folate antagonist methotrexate (MTX) have a well-documented longterm efficacy in maintaining remission. ${ }^{6-10}$ Immunosuppression with AZA/6-MP is, however, of limited value for induction of remission, and fewer than half of the patients sensitive or resistant to steroids show long-term benefit. ${ }^{6-10}$ AZA or 6-MP treatment is also associated with an important risk of side effects.
The introduction of infliximab (IFX), a mousehuman chimeric immunoglobulin G1 (IgG1) monoclonal antibody to tumour necrosis factor $\alpha$ $(\mathrm{TNF} \alpha)$ (Remicade Centocor, Malvern, Pennsylvania, USA) has offered us new treatment options in CD. IFX has been demonstrated in several studies to be very efficacious for the treatment of refractory luminal and fistulising CD. ${ }^{11-14}$ In the short term, up to $80 \%$ of patients experience a rapid improvement of their symptoms and up to $50 \%$ of patients have a complete remission. ${ }^{11-14}$ With scheduled 8 weekly maintenance treatment, IFX not only maintains disease improvement ${ }^{1213} 15$ but also induces mucosal healing in nearly half of the treated patients. ${ }^{16}$ Data from the ACCENT I and II studies also show that scheduled treatment with IFX decreases the rate of hospitalisations and surgical interventions. ${ }^{13} 15$

Although IFX has now been used in clinical practice for the treatment of CD for almost 10 years, there are few data published on its long-term efficacy beyond 1 year and on the durability of the effects of the drug and its impact on the long-term outcome of CD.

Here we report on the real-life experience and the long-term outcome of IFX treatment in a consecutive series of 614 patients with CD treated at our referral centre. We specifically looked at maintenance of improvement, the ability to discontinue steroids, and the need for hospitalisations and surgery in comparison with the pre-IFX period. All patients had been followed according to a prospectively planned follow-up protocol.

\section{MATERIALS AND METHODS}

\section{Patients and treatment}

A total of 761 patients with IBD (614 patients with CD, 139 patients with ulcerative colitis and 8 patients with indeterminate colitis) received their first IFX dose between November 1994 and January 2007 at the University Hospital of Leuven.

Long-term follow-up until the end of March 2007 was completed in all 614 patients with CD. The analysis of this study focused on the patients who responded to the drug within 10 weeks - that is, 547 patients

The majority of patients with CD were treated for luminal CD ( $n=420,68 \%), 170$ patients were treated for fistulising CD (28\%) and in $24 \mathrm{CD}$ patients the main indication was extraintestinal manifestations (4\%). Treatment was started on an episodic basis in 353 patients (64.5\%), and 194 patients $(35.5 \%)$ received scheduled treatment 
every 8 weeks from the start (fig 1). In 163 patients (29.8\%) who received episodic treatment the regimen had to be switched to scheduled treatment due to loss of response after a median of 26 months (interquartile range (IOR) 12-47) and a median of six infusions (IOR 4-10) (fig 1). Most patients in the early years were treated episodically, which reflects patterns of clinical practice and local reimbursement policies at that time. Overall, 55 patients (8.96\%) of the total cohort of 614 patients with CD had received IFX in different clinical studies. Only infusions with active IFX during the study and open infusions during follow-up were included in our analysis.

Patients' baseline characteristics are listed in table 1.

\section{Primary analysis}

This is an observational study of a single-centre cohort where a prospectively designed standardised follow-up schedule was used for the follow-up of all patients by experienced clinicians. The primary analysis looked at what proportion of patients with an initial response to IFX showed sustained clinical benefit on IFX treatment throughout their entire follow-up.

A secondary analysis concerned the number of interventions needed to keep the disease under control in the long term, the proportion of patients who had to stop IFX because of loss of response or side effects, and the proportion of patients who could discontinue IFX because they were in remission.

Another goal of this study was to identify predictors for sustained clinical benefit.

Furthermore, we analysed the rate of surgery and hospitalisations throughout the period after initiation of treatment with IFX in comparison with the rates prior to the start of this treatment.

\section{Definitions}

Sustained clinical benefit was defined as a lasting control of the disease activity during follow-up with persistent improvement of the symptoms. In patients treated episodically, relapse of CD symptoms triggered a repeated administration of IFX.
IFX treatment was defined as episodic (on demand) if IFX was given only upon relapse of symptoms and it was defined as scheduled if IFX was intentionally planned every 8 weeks.

Interventions included all therapeutic options involving dose increases of IFX necessary to keep the disease under control or to regain control, and included any shortening of the interval between IFX infusions (up to a minimum of 4 weeks) and/or an increase of the dose of IFX (maximum dose $10 \mathrm{mg} / \mathrm{kg}$ ) as well as a change from episodic treatment to scheduled treatment with administration of the IFX infusions every 8 weeks.

Any resection of a part of the gut, or strictureplasty for stenosing, or fistulising complications and faecal diversion (ostomy) were considered as major abdominal surgery (MAS). The rates were calculated per 100 patient years. Procedures such as abscess drainage or endoscopic dilatations were not counted.

Acute infusion reactions were defined as any adverse events that occurred during or within $1 \mathrm{~h}$ after the infusion of IFX. All reactions occurring later were considered delayed infusion reactions. These were defined as serum sickness-like reactions when there was occurrence of myalgia, arthralgia, fever or rash, or any combination of these symptoms. Severe adverse events (SAEs) were defined as any adverse event that resulted in hospitalisation or prolonging of hospitalisation, was fatal or life threatening or led to significant disability.

\section{Administration of IFX and assessment of initial response to IFX} The initial response to IFX was assessed by experienced clinicians at 4 or 10 weeks after a single infusion or loading schedule, respectively. Patients who became completely symptom free were considered full responders to the IFX treatment. Patients who had distinct clinical improvement with an obvious decrease of the disease activity but who still had symptoms were considered partial responders. Patients who had no benefit after a median of two infusions discontinued treatment and were considered primary non-responders.

Biological activity was also assessed in all the patients using C-reactive protein (CRP) levels at baseline and week 4 in the
Figure 1 Schedule of treatment with infliximab (IFX) in 614 patients with Crohn's disease (CD). *After a median of 26 months (interquartile range (IOR) $12-47$ ) and a median of six infusions (IQR 4-10).

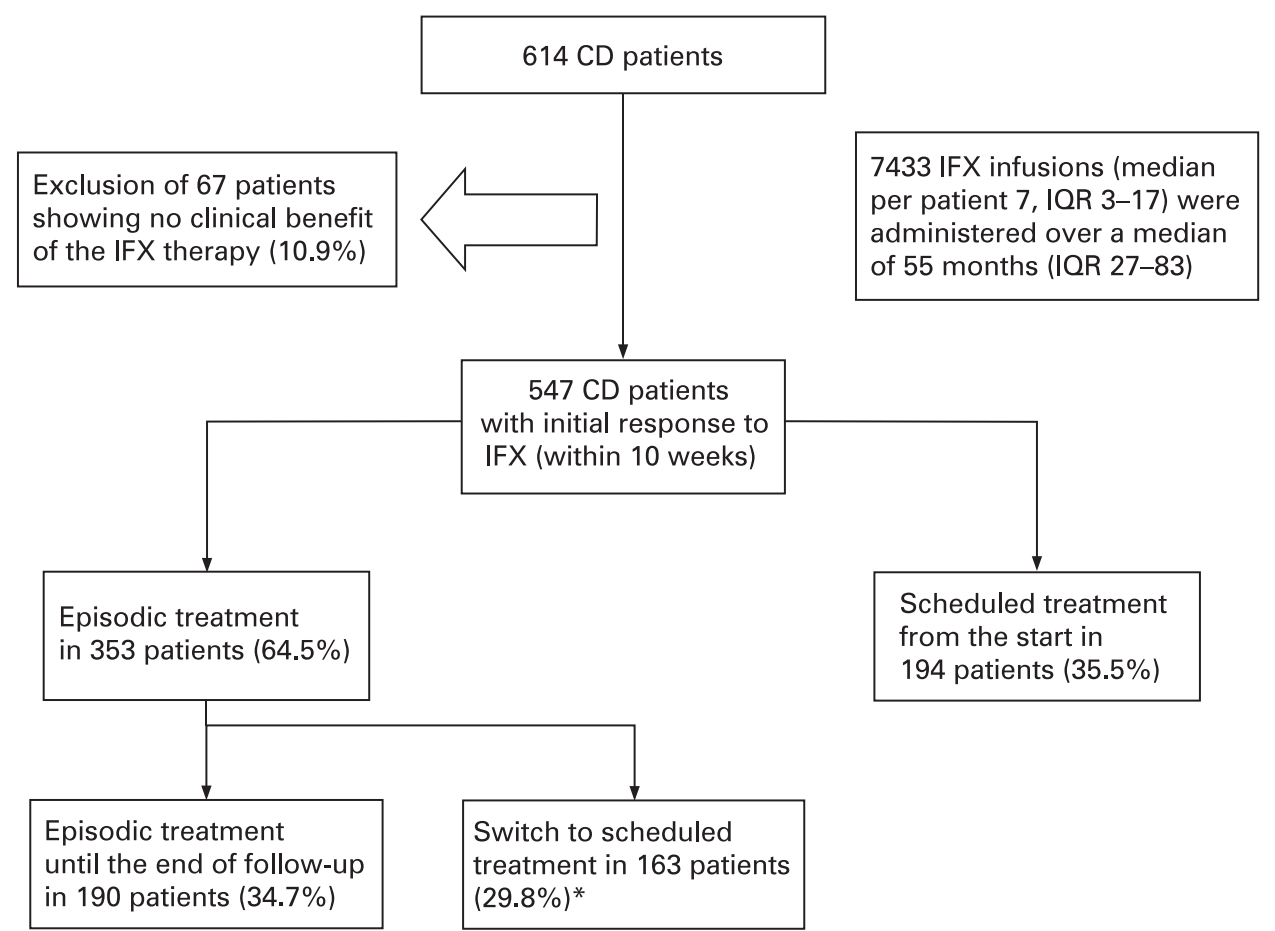


Table 1 Patients' baseline characteristics

\begin{tabular}{|c|c|}
\hline Male/female (\%) & $240 / 374(39 / 61)$ \\
\hline \multicolumn{2}{|l|}{ Indication for IFX treatment (\%) } \\
\hline Luminal CD & $420(68)$ \\
\hline Fistulising CD & $170(28)$ \\
\hline Luminal $C D$ and mainly extraintestinal manifestations & $24(4)$ \\
\hline Median (IQR) age at diagnosis (years) & $22.8(17.8-29.9)$ \\
\hline Median (IQR) age at first infusion (years) & $33.9(24.9-43.3)$ \\
\hline Median (IOR) duration of disease prior to first IFX (months) & $92.1(28.2-188.9)$ \\
\hline Median (IOR) patient's follow-up of IFX treatment (months) & $55.3(27.4-83.0)$ \\
\hline \multicolumn{2}{|l|}{ Location of disease (\%) } \\
\hline Ileitis & $103(16.8)$ \\
\hline Colitis & $148(24.1)$ \\
\hline Ileocolitis & $326(53.1)$ \\
\hline Upper GIT & $37(6.0)$ \\
\hline Smokers $(\%)(n=423)$ & $202(47.8)$ \\
\hline \multicolumn{2}{|l|}{ Concomitant medication at first IFX (\%) } \\
\hline Aminosalicylates & $202(32.9)$ \\
\hline Steroids & $198(32.2)$ \\
\hline 6-MP/AZA & $305(49.7)$ \\
\hline MTX & $58(9.4)$ \\
\hline Median (IOR) baseline CDAI $(n=362)$ & $253(175-336)$ \\
\hline Median (IOR) baseline CRP $\mathrm{mg} / \mathrm{l}(\mathrm{n}=481)$ & $12.9(5.0-32.4)$ \\
\hline Previous major abdominal surgery (\%) & $238 / 614(38.8)$ \\
\hline
\end{tabular}

case of a single infusion and at week 10 in the case of induction treatment at weeks 0,2 and 6 .

A drop in CRP levels of $>50 \%$ between baseline and assessment time or normalisation of the CRP levels $(<3 \mathrm{mg} / \mathrm{l})$ was classified as a biological response.

\section{Statistical analysis}

In this longitudinal observational cohort study, the SAS 9.1 statistical software package (SAS Institute, Cary, North Carolina, USA) and the SPSS 15.0 software (SPSS, Chicago, Illinois, USA) were used to perform all appropriate statistical analyses. Medians with the IOR were calculated for continuous data and percentages were computed for discrete data. The SAS procedure GENMOD was used to analyse count data. The sustained clinical benefit of IFX was estimated by Kaplan-Meier analyses in SPSS. These were verified and complemented by analyses in SAS, adopting the procedure LIFETEST and procedure PHREG for Cox proportional hazards analysis. To compare hazard rates in populations defined by one variate at a time, both the Breslow and the Logrank tests were used.

\section{RESULTS}

Over a median of 4.6 years (IOR 2.3-6.9) or 55 months (IOR 27-83) a total of 7433 IFX infusions were administered to 614 patients with $\mathrm{CD}$ (fig 1). Patients receiving episodic IFX treatment throughout $(n=190)$ received overall 1009 IFX infusions during a median follow-up of 5.9 years (IOR 4.0-7.2) with a median of 0.8 infusions (IOR 0.4-1.3) and a median total dose of $260 \mathrm{mg}$ of IFX per patient year (IOR 131-452). Patients with a switch from episodic to scheduled treatment in the long term $(n=163)$ received overall 3806 infusions during a median follow-up of 6.2 years (IOR 4.5-7.4) with a median of four infusions (IOR 3-5) and a median total dose of $1456 \mathrm{mg}$ of IFX per patient year (IOR 757-1794). Patients receiving scheduled treatment from the start $(n=194)$ received overall 2442 infusions during a median follow-up of 1.9 years (IOR 0.9-3.4) and with a median of seven infusions (IOR 4-8) and a median total dose of $2134 \mathrm{mg}$ of IFX per patient year (IOR 1298-2679), respectively.

\section{Initial response to IFX}

Overall, 547 (89.1\%) patients with CD had a clinical improvement of their symptoms after the initiation of the IFX treatment. A total of 295 patients (48.1\%) showed partial response and $41.0 \%(n=252)$ had full response. A total of 67 patients $(10.9 \%)$ had no initial response to IFX, and IFX was discontinued early after a median of two infusions (IOR 2-3) (figs 1 and 2).

CRP levels at baseline and at follow-up were available in 481 patients with CD with an initial response to IFX. The majority of these patients had an increased CRP level at baseline $(>3 \mathrm{mg} / \mathrm{l}$, $\mathrm{n}=401,83.4 \%$ ). The median CRP level at baseline was $12.9 \mathrm{mg} / \mathrm{l}$ (IOR 5.0-32.4) and decreased to $2.1 \mathrm{mg} / \mathrm{l}$ (IOR 1.5-7.3) at followup at week 4 or 10. A total of 309/401 patients (77.1\%) responded biologically to IFX, including 186 patients (46.4\%) displaying a decrease in CRP back to normal values $(<3 \mathrm{mg} / \mathrm{l})$ and 123 patients (30.7\%) with a drop in CRP of $>50 \%$. Eighty patients (80/481, $16.6 \%)$ already had normal CRP levels $(<3 \mathrm{mg} / \mathrm{l})$ at baseline. Ninety-two patients $(92 / 401,22.9 \%)$ showed no CRP decrease.

\section{Sustained clinical benefit long term}

At the end of follow-up in March 2007, 347 of the 547 initial responders to IFX (63.4\%) showed sustained clinical benefit from IFX (figs 2, 3A and 4A). Of these, 237 patients (43.3\%) were still receiving IFX with sustained control of disease activity, while in $110 \mathrm{CD}$ patients $(20.1 \%$, 63 with luminal $\mathrm{CD}, 42$ with fistulising $\mathrm{CD}$ and 5 mainly with extraintestinal manifestations), IFX treatment had been stopped after a median of 6.2 months (IOR 1.4-16.6) and a median of four infusions (IOR 2-7), while the patients were in remission. They stayed in remission for a median of 47.3 months (IOR 20.8-66.4) after stopping IFX treatment.

The great majority of these 110 patients, namely 81 (73.6\%), were treated episodically with IFX and did not receive retreatment because they stayed in remission. Nineteen (17.3\%) had been on scheduled treatment and 10 (9.1\%) had been switched from episodic to scheduled treatment and IFX was discontinued because of the doctor's or patient's decision. After stopping the IFX treatment, 92 of the 110 patients (84\%) continued on IMMs (75 patients with AZA, 17 with MTX). A total of 67 of these patients (61\%) were already receiving IMMs at the start of IFX, whereas in 25 patients, IFX and AZA $(\mathrm{n}=20)$ or MTX $(\mathrm{n}=5)$ had been started simultaneously $(23 \%)$ and IFX was used as a bridging treatment and was stopped after a median of two infusions (IOR 1-3). Eighteen patients had received IFX without any concomitant IMM treatment and were able to stop IFX while in remission without any maintenance treatment.

\section{Discontinuation of IFX treatment}

Overall, 200/547 patients (36.6\%) stopped IFX, due to loss of response $(n=118,21.6 \%)$, side effects $(n=70,12.8 \%)$ or for other reasons ( $n=12,2.2 \%$ ) (fig 2 ).

A total of $39.7 \%$ of patients who had started on episodic treatment (140/353) had to stop IFX treatment compared with $24.7 \%$ of patients (48/194) receiving scheduled treatment from the start. While the proportion of patients who had to stop IFX 


\section{CD patients with initial response to IFX}

IFX treatment stopped in 70 patients

due to side effects $(12.8 \%)$

IFX treatment stopped in 118 patients

due to loss of response $(21.6 \%)$

IFX treatment stopped in 12 patients for other reasons $(2.2 \%)$

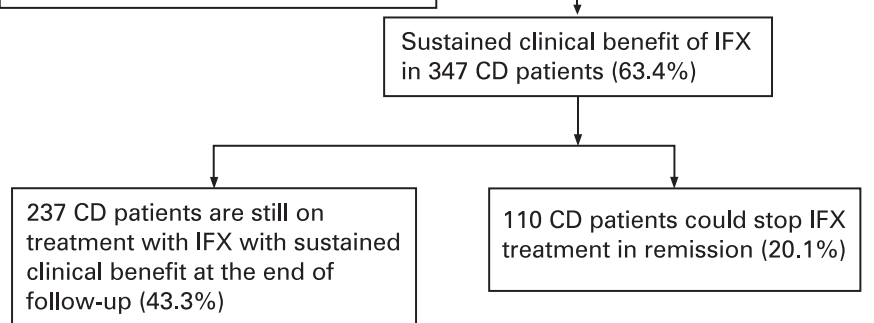

Figure 2 Outcome of treatment of patients with Crohn's disease (CD) with infliximab (IFX) and reasons for its discontinuation.

due to loss of response was comparable for both groups $(22.9 \%$ $(n=81)$ in the episodic group vs $19.1 \%(n=37)$ in the scheduled group; $p=0.3286$, Fisher exact test), significantly more patients in the group treated episodically had to stop because of side effects as compared with patients receiving scheduled treatment from the start $(n=59 / 353, \quad 16.7 \%$ vs $n=11 / 194, \quad 5.6 \%$; $p=0.0001$, Fisher exact test).

The median time to drop-out from IFX treatment was 79.1 months (IOR 67.1-91.0) for the patients who started on episodic treatment $(n=353)$. Since the median survival time is defined as the lowest survival time for which the survivor function is $\leqslant 50 \%$, and since the survivor function for the scheduled treated group $(n=194)$ does not fall below $50 \%$, the median time to drop-out from IFX for the scheduled group cannot be estimated. Thus, only the mean time to drop-out from IFX can be given for this group, namely 64.8 months (range 57.0-72.6). Although the presented estimated mean survival times do take into account censoring, they are underestimates of the true means. The median drop-out rate from treatment per year was 10.7\% (IOR 7.1-14.3) for the episodic group and 7.1\% (IOR 0.0-8.6) for the scheduled group.

\section{Discontinuation of IFX treatment due to side effects}

In 70 of $547 \mathrm{CD}$ patients, IFX treatment was discontinued because of side effects after a median of five infusions (IOR 3-11) and after a median duration of 29.5 months (IOR 8.3-53.0). These included acute infusion reactions in 15 patients $(15 / 547,2.7 \%)$ and delayed infusion reactions in $33 \mathrm{CD}$ patients $(33 / 547,6.1 \%)$. Other SAEs occurred in 22 patients with CD (22/547, 4.0\%): four patients had to stop IFX due to serious acute infusion reactions and 9 patients because of serious delayed infusion reactions. Serious infections were diagnosed in 5 patients, including an infection with herpes zoster, an abdominal tuberculosis infection and an Aspergillus infection. The Aspergillus infection was lethal and was considered to be directly related to the IFX treatment. One sudden death occurred during the IFX treatment but was not considered to be related to the IFX treatment. Three patients had to stop IFX because of neurological complications (one patient with a not proven neuritis optica, one patient with a diagnosed central demyelinising lesion and one patient with extensive multiple sclerosis-like neurological symptoms).

Discontinuation of IFX treatment due to loss of response to IFX A total of $118 \mathrm{CD}$ patients (21.6\%) presented with a loss of response to treatment with IFX and failure of dose increase interventions. IFX treatment was stopped in these patients after a median of eight IFX infusions (IOR 4-14) and after having been receiving treatment with IFX for a median of 21.5 months (IOR 8.8-42.4). Sixty patients ( $\mathrm{n}=60 / 118,50.8 \%$ ) were either switched to adalimumab (Humira, Abbott Laboratories North Chicago, Illinois, USA) $(n=50 / 118,42.4 \%)$ or they were included in studies with the pegylated anti-TNF antibody fragment certolizumab (Cimzia, UCB Group, Brussels, Belgium) $(n=10 / 118,8.5 \%)$. Twelve patients were included in studies with other biological agents $(10.2 \%), 32$ patients with CD (27.1\%) underwent surgery and 14 patients $(11.9 \%)$ received rescue treatment, mainly with steroids.

Of the 547 initial responders, 12 patients (2.2\%) discontinued IFX treatment for different reasons, including pregnancy, moving abroad, patient's preference, problems with funding, diagnosis of breast cancer and relapse of hepatitis B after a median of three infusions (IOR 3-7) and a median of 15.2 months (IOR 5.9-31.0).

\section{Mortality during IFX treatment}

Ten patients died during the median follow-up of 55 months. The median age at death was 58 years (range 35-83). Seven patients died after they had already stopped IFX for a median of 29 months (range 5-66). One patient died during IFX treatment due to an Aspergillus infection, and one sudden death occurred. In a 45-year-old woman a pancreatic carcinoma was diagnosed 1 month after her last IFX infusion and she died 5 months after the diagnosis and outside of the observational period of our study. The death due to Aspergillus infection was considered to be directly related to IFX treatment; three deaths were considered possibly related and six deaths were unrelated.

\section{Need for interventions during IFX treatment}

Of the 547 initial responders, $50 \%(\mathrm{n}=273)$ did not need any intervention, whereas $26 \%(n=143)$ needed one intervention, $10 \%(n=56)$ needed two and $14 \%(n=75)$ needed $\geqslant 3$ interventions. Of the patients who were started on episodic IFX treatment ( $n=353$, fig 1), $163(46 \%)$ had to switch to treatment every 8 weeks after a median of 26 months (IOR 12-47) and/or six infusions (IOR 4-10) (fig 1).

A reduction of the interval between the infusions became necessary in 108 patients (19.7\%), an increase of the dose to $10 \mathrm{mg} / \mathrm{kg}$ and/or a re-induction with IFX infusions at weeks 0,2 and 6 in 144 patients $(26.3 \%, 63$ patients with a re-induction and 89 patients with an increase of the dose of IFX) or an increase of the dose plus a reduction of the interval in only 21 patients (3.8\%). Overall, 103 of the 144 patients (71.5\%) with an increase in dose and/or a re-induction with IFX were able to go back to the standard dose of $5 \mathrm{mg} / \mathrm{kg}$, and $61.9 \%$ of patients (13/ 21) with an increase of dose and a shortening of the interval were able to go back to $5 \mathrm{mg} / \mathrm{kg}$ and treatment every 8 weeks. A total of $28.7 \%$ of patients with a shortened interval between the infusions $(31 / 108)$ could again extend the interval to 8 weeks. In the total cohort of initial responders, only $21.6 \%(n=118)$ had to stop IFX because of loss of response, despite interventions. 
A

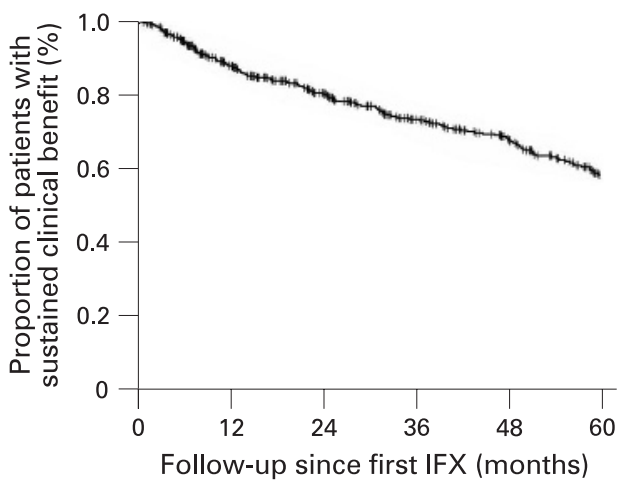

$\begin{array}{llllll}\text { At risk: } & 547 & 414 & 330 & 253 & 201\end{array}$

C

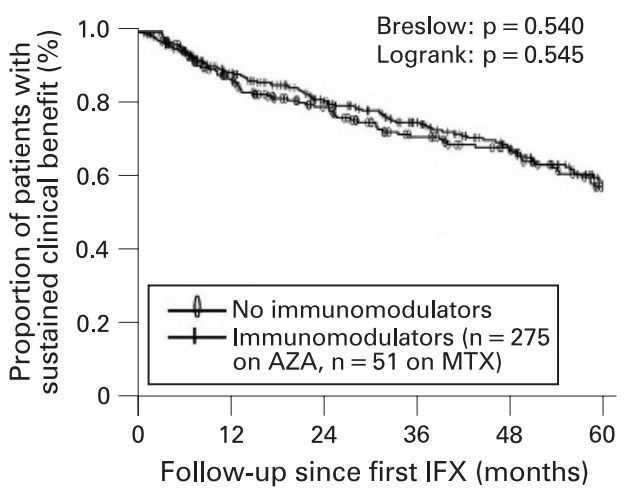

$\begin{array}{lllllrr}\text { At risk: } & 221 & 166 & 133 & 104 & 88 & 62 \\ & 326 & 248 & 197 & 149 & 113 & 75\end{array}$

E

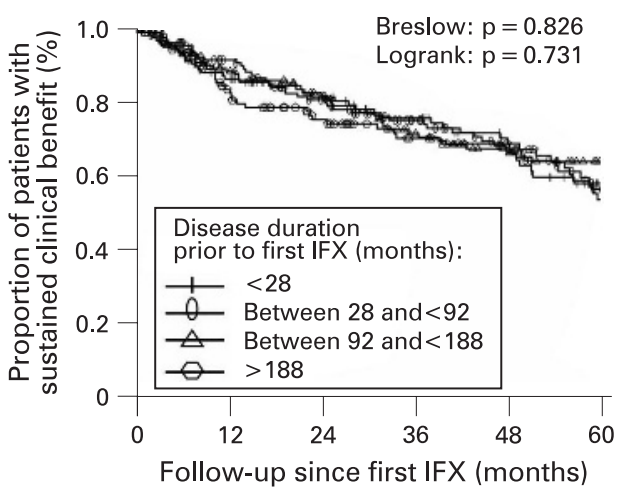

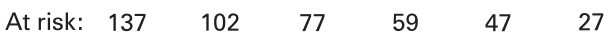

$\begin{array}{rrrrrr}137 & 102 & 77 & 59 & 47 & 27 \\ 141 & 113 & 92 & 71 & 53 & 34 \\ 135 & 112 & 94 & 72 & 58 & 45 \\ 134 & 87 & 67 & 51 & 43 & 31\end{array}$

B

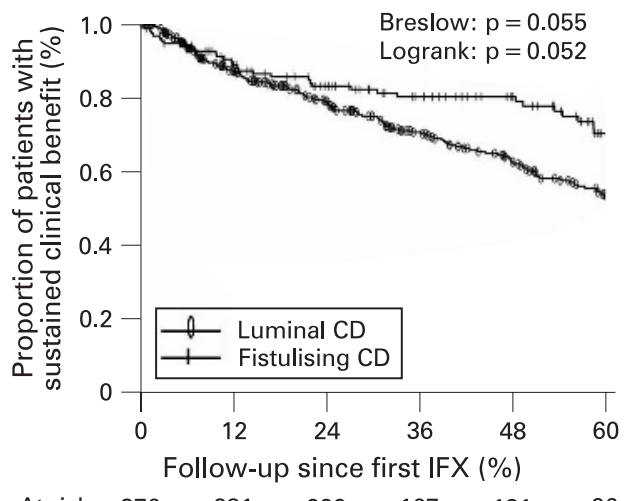

$\begin{array}{lrrrrrr}\text { At risk: } & 376 & 281 & 222 & 167 & 131 & 96 \\ & 152 & 118 & 95 & 78 & 65 & 40\end{array}$

D

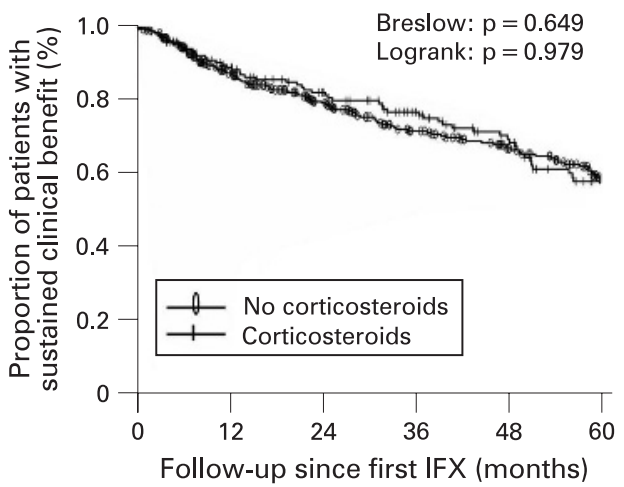

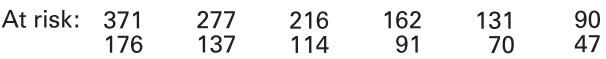

$\mathrm{F}$

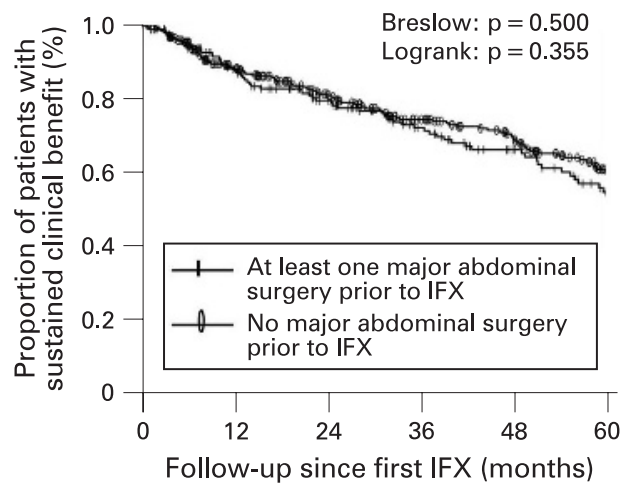

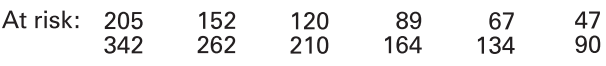

Figure 3 Sustained clinical benefit of infliximab (IFX) in patients with Crohn's disease (CD) who initially responded to the IFX treatment in the overall cohort $(A)$ and stratified by indication for the IFX treatment $(B)$, concomitant treatment with immunomodulators at baseline (C), concomitant treatment with corticosteroids at baseline (D), duration of the disease prior to the IFX treatment (E) and history of major abdominal surgery prior to the IFX treatment (F). AZA, azathioprine; MTX, methotrexate.

\section{Steroid tapering during IFX treatment}

A total of 198 patients received corticosteroids at baseline $(32.2 \%)$. The majority ( $\mathrm{n}=141,71.2 \%$ ) could definitively stop corticosteroid treatment after a median of 12 weeks (IOR 5-36) and a median of two IFX infusions (IOR 1-3) with no need for re-treatment (fig 5). The proportion of patients who were able to stop was significantly higher in the group receiving scheduled treatment and in the group of patients who were switched to scheduled treatment than in the group who received episodic treatment throughout $(p=0.024$, fig 5$)$. Also the time to complete withdrawal of corticosteroids was shorter in patients who were treated every 8 weeks with IFX (median 8.7 weeks, IOR 5-26), as compared with patients who started IFX episodically (median 15 weeks, IOR 6-51) ( $p=0.057$, Mann-Whitney U test). In contrast, only $27.3 \%(6 / 22)$ of patients with no short-term clinical benefit of IFX treatment were able to stop corticosteroids (Logrank: $p<0.0001$, fig 5). 
Figure 4 Sustained clinical benefit of infliximab (IFX) in patients who initially responded to the IFX treatment and stratified by treatment regimen $(A)$, baseline $\mathrm{C}$-reactive protein (CRP) levels (B) and drop of CRP levels in patients with an increased baseline CRP level (C). Overall, $76.25 \%$ of patients with normal baseline CRP levels received concomitant immunomodulators (IMMs) at baseline $(\mathrm{n}=61,56$ received azathioprine (AZA) and 5 received methotrexate $(\mathrm{MTX}))$, and 31 of them were also treated with corticosteroids (CS) (38.75\%). A total of $55.11 \%$ of patients with elevated baseline CRP levels received concomitant IMMs at baseline ( $n=221,187$ patients AZA and 34 patients MTX) and $30.92 \%(n=124)$ of them received CS at baseline.
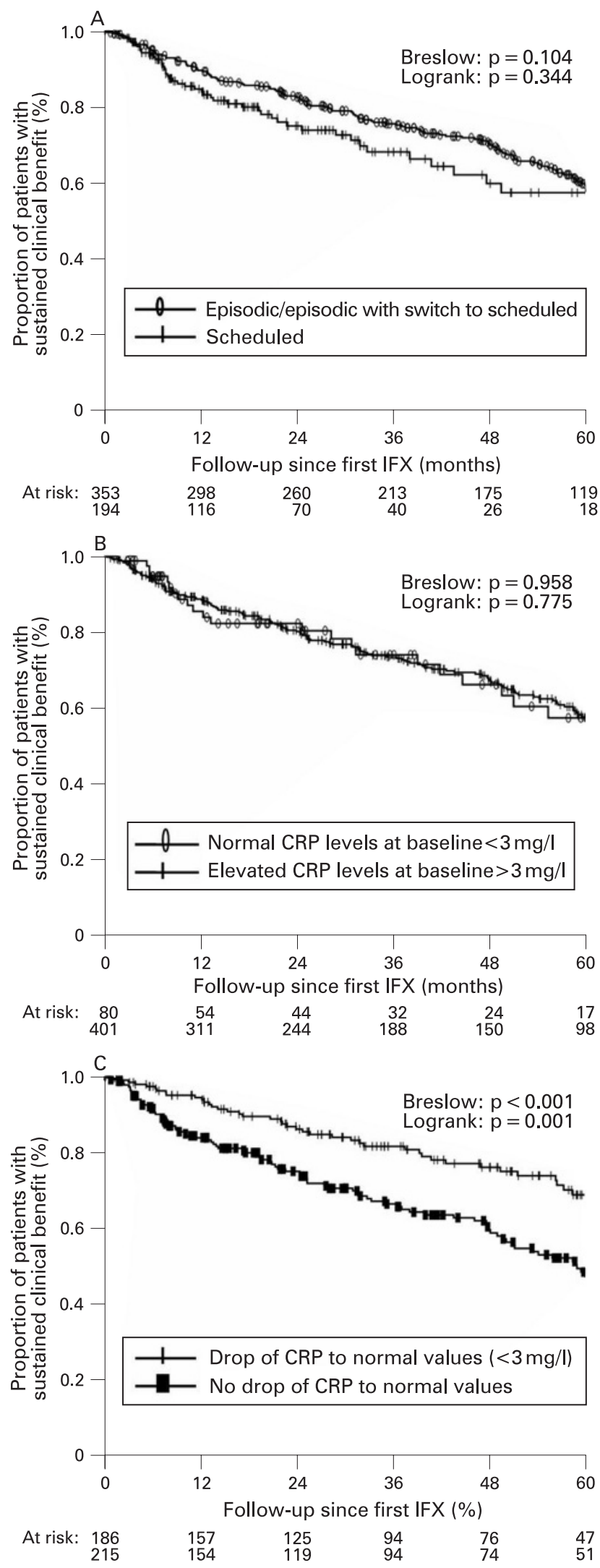

\section{Withdrawal of IMMs during IFX treatment}

Half of the patients were on AZA at baseline ( $\mathrm{n}=305,49.7 \%)$ and 58 patients $(9.4 \%)$ were on MTX. One-third of patients on AZA at baseline $(n=104,34.1 \%)$ stopped AZA after a median of 15 months (IOR 6-31) and a median of six infusions (IOR
$3-11)$. Of these, 61 patients (58.7\%) stayed off immunosuppression until the end of follow-up, and 43 patients (41.3\%) were switched to concomitant MTX treatment. Half of the patients on MTX at baseline $(n=28,48.3 \%)$ stopped MTX after a median of 14 months (IOR 6-33) and a median of four infusions 
(IOR 3-6), 82.1\% ( $\mathrm{n}=23,82.1 \%)$ stayed off IMMs until the end of follow-up, and five patients (17.9\%) were switched to concomitant AZA. Overall, 116 of the 363 patients (32\%) who received IMMs at the start were able to stop the IMM treatment after a median of 25 months (IOR 11-53) and a median of six infusions (IOR 3-12) with no need for retreatment in the long-term.

\section{Hospitalisations and surgery during IFX treatment}

A hospitalisation rate of $42.3 \%(260 / 614)$ was observed after starting IFX. More patients started on episodic treatment required hospitalisation $(46.7 \%, 165 / 353$, median follow-up 72.6 months, IOR 50.9-87.1) compared with patients receiving scheduled treatment from the start $(25.7 \%, 50 / 194$, median follow-up 22.9 months, IOR 11.0-41.1; $\mathrm{p}<0.001$ ). The number of hospitalisations per patient was also higher in those receiving episodic treatment (median 2, IOR 1-4) than in patients on scheduled treatment (median 1, IOR 1-2). The highest hospitalisation rate of $67.2 \%(45 / 67)$ was observed in patients who had to stop IFX prematurely because of no clinical benefit of the treatment.

A total of $23.5 \%(144 / 614)$ of patients needed MAS during IFX treatment. Initial response to IFX and sustained clinical benefit of IFX up to the end of follow-up were associated with a significant decrease in the need for MAS after the initiation of the IFX treatment $(p<0.001$, fig $6 A)$. Primary non-response to IFX or loss of response were associated with a significant increase in the need for MAS $(p<0.001$, fig 6A). Patients with scheduled infusions every 8 weeks from the start and patients who were switched from episodic to treatment every 8 weeks with IFX had a significantly lower need for MAS in the long term compared with patients with an episodic treatment regimen until the end of follow-up (Logrank: $p<0.020$, fig $6 \mathrm{~B}$ ).

\section{Predictors of the long-term outcome of the IFX treatment}

A decrease in elevated baseline CRP levels back to normal values $(<3 \mathrm{mg} / \mathrm{l})$ after initiation of IFX treatment was significantly associated with a better outcome of IFX treatment in the long term (Logrank: $p=0.001$, fig 4C).

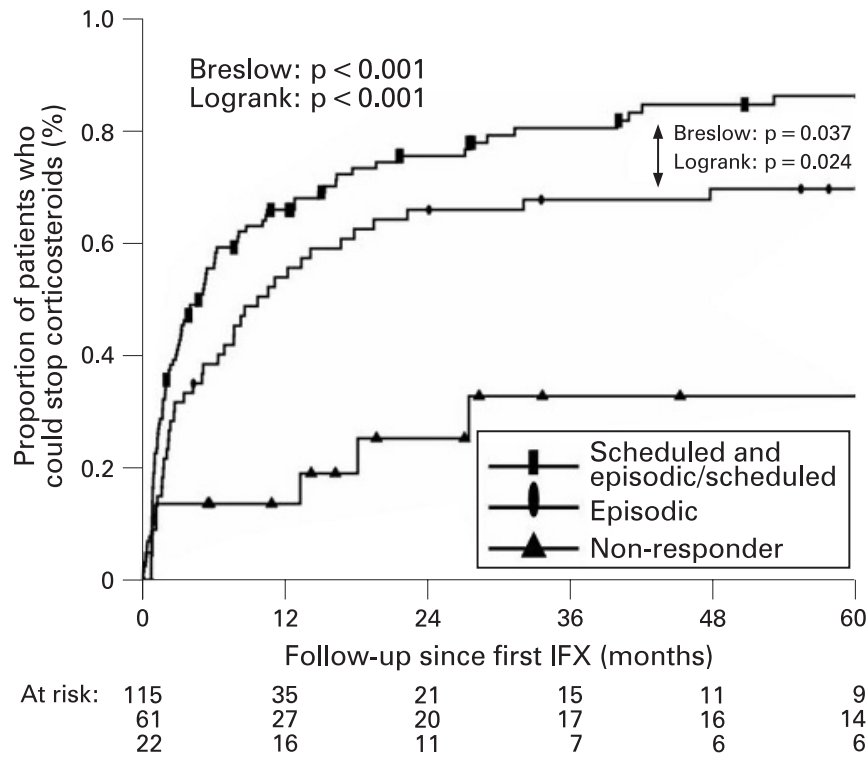

Figure 5 Ability to wean patients off steroids in those treated with corticosteroids at baseline stratified by the initial response to infiximab (IFX) and by treatment schedule.
Absence of short-term clinical benefit of IFX treatment was associated with a significantly greater need for MAS and with decreased ability to stop corticosteroid treatment in the long term (Logrank: $p<0.001$, figs 5 and $6 \mathrm{~A}, \mathrm{~B}$ ).

Other factors including the indication for IFX treatment, concomitant treatment with IMMs or with corticosteroids at baseline, disease duration prior to the initiation of the IFX treatment, prior history of MAS or CRP level at baseline did not have an influence on the outcome of IFX treatment in the long term (figs $3 \mathrm{~B}-\mathrm{F}$ and $4 \mathrm{~B}$ ).

\section{DISCUSSION}

To date, there are only limited data available on the long-term outcome of IFX treatment for CD in clinical practice. A few studies reported on a durable efficacy of IFX also beyond 1 year, ${ }^{17-19}$ but the size of the cohorts was small, the follow-up limited and not all patients treated were followed long term. The present study assessed the long-term efficacy of IFX treatment in a large cohort of patients with CD with a median follow-up of almost 5 years. All patients who have been treated with IFX between November 1994 and March 2007 were included in our study and were followed in a prospectively designed follow-up schedule.

The short-term response rate in this study (within the first 10 weeks) is higher than that reported in the initial randomised placebo-controlled trials and in the ACCENT I and II trials. ${ }^{11-14}$ This is without doubt due to the lack of well-defined criteria for (non-) response using the Crohn's Disease Activity Index (CDAI) so that all patients who showed some clinical benefit were allocated to scheduled treatment. In a small study, Pearce et al found comparable initial response rates. In that study, $75 \%$ of patients with luminal CD responded at 8 weeks to a single dose of IFX as did $92.9 \%$ of patients with IFX at 0,2 and 6 weeks. ${ }^{20}$ In comparison, the response rates in our study at week 10 for patients who received induction infusions at weeks 0,2 and $6(29.6 \%, \mathrm{n}=182)$ and for patients who received a single infusion $(70.4 \%, \mathrm{n}=432)$ were not significantly different (92.3\% response rate to IFX for patients with an induction scheme vs $87.7 \%$ for patients with a single infusion, $p=0.12$, Fisher exact test).

The present analysis shows excellent efficacy of IFX treatment to maintain improvement not only during 1 year as in the published trials, ${ }^{11-14}$ but also during a median follow-up of 4.6 years. Indeed, almost two-thirds of the initial responders to IFX in our cohort (63.4\%) maintained benefit until the end of their follow-up. The efficacy of treatment was similar for luminal and fistulising disease, and other baseline characteristics, such as concomitant immunosuppression, duration of disease and prior surgery did not influence outcome.

The drop-out rate from IFX treatment was not different for patients started on episodic treatment compared with patients receiving scheduled treatment from the start. However, patients in the episodic group always needed to suffer a relapse of symptoms before they were given a repeat infusion. On the other hand, episodic treatment allowed us to identify an important proportion of patients who could discontinue treatment without suffering a relapse. The majority of these patients remained on immunosuppression.

The total dose of IFX administered was very low in patients who stayed on episodic treatment throughout. There was not much difference in total dose between patients who were started on scheduled treatment and patients who had to switch from episodic to scheduled treatment. 

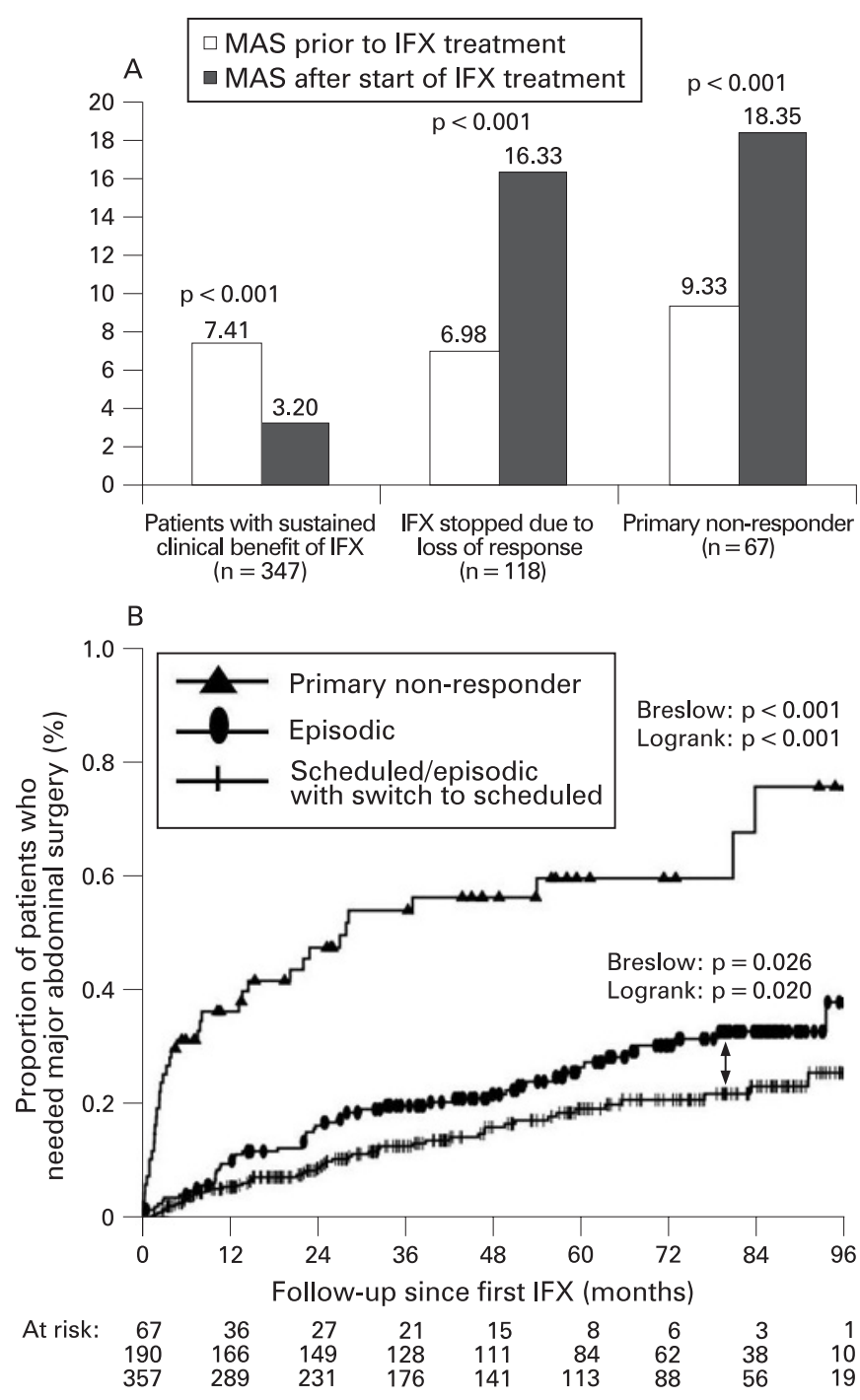

Figure 6 Need for major abdominal surgery (MAS) in infliximab (IFX)treated patients. The number of MAS per 100 patient years before initiation of IFX as compared with after the start of IFX and stratified by long-term response to IFX (A), and timetable of proportions of patients needing MAS stratified by treatment schedule and response to IFX treatment (B).

We could not identify predictors for a long-lasting response in our episodic cohort.

Given their important side effects in the long term, corticosteroid sparing is one of the most important treatment aims in the long-term treatment of CD. The ACCENT I study demonstrated the steroid-sparing effect of IFX over a 1 year period. ${ }^{12}$ In the present cohort, steroid withdrawal was possible in $>70 \%$ of patients taking steroids at baseline, which is more than reported in the ACCENT I study. In our study, these patients also stayed off steroids during their further follow-up.

Patients who received scheduled treatment from the start or were switched from episodic to scheduled treatment were able to stop steroids more frequently and earlier than patients who were treated episodically throughout.

The ACCENT I and ACCENT II studies showed that scheduled treatment with 8 weekly IFX in responders is associated with a lower need for hospitalisations and surgical interventions over a follow-up period of 1 year compared with episodic treatment. ${ }^{12} 15$ Our study shows that the outcome of
$\mathrm{CD}$ is also altered over the course of several years. The rate of hospitalisations and surgery was lowest in the patients who were treated in a scheduled regimen and the rate of surgery was significantly decreased in comparison with the rate in the same patients prior to initiation of IFX. In contrast, patients who did not respond to IFX or who lost response and discontinued IFX often needed surgery, and the rate of surgical interventions in these patients was significantly increased in comparison with that before starting IFX.

Treatment with IFX was safe in our patients. Side effects forced a discontinuation of the IFX treatment in $13 \%(n=70)$ of the initial responders to IFX. The majority of them had to stop IFX because of delayed infusion reactions $(n=33,6 \%)$ or uncontrolled acute infusion reactions ( $\mathrm{n}=15,3 \%$ ). SAEs occurred in 22 patients (4\%). There was one certain drug-related mortality case and three deaths that were possibly related to IFX treatment. More details about the safety profile of IFX in the whole cohort of patients with IBD treated with IFX at our IBD unit are the subject of a separate study by Fidder et al. ${ }^{21}$

\section{CONCLUSION}

The majority of patients with CD treated with IFX (63.4\%) in this cohort showed sustained clinical benefit during a median follow-up of almost 5 years. Although episodic treatment was effective, more than half of the patients who started with episodic treatment needed to be switched to scheduled treatment. Moreover, scheduled treatment was more effective than episodic treatment for steroid withdrawal and avoidance of hospitalisations and surgery. We feel therefore that scheduled treatment should be preferred for optimal disease control in patients with CD. However, one in three patients who are started on episodic ITX can discontinue therapy while staying in remission with continued immunosuppression treatment, but we could not identify predictors for this favourable outcome. To date, no rules regarding stopping treatment have been identified in patients treated with scheduled IFX.

This study reflects real-life experience with IFX treatment in $\mathrm{CD}$ in daily clinical practice. The excellent long-term outcome in the present cohort may be explained by careful patient selection. Close standardised monitoring of clinical symptoms and laboratory findings in the patients during follow-up at regular scheduled visits allowed optimal adjustment of the IFX treatment to the disease activity, which led to improved longterm outcome.

Acknowledgements: FS received an ECCO (European Crohn's and Colitis Organisation) fellowship and an educational grant from Centocor bv.

Competing interests: None.

\section{REFERENCES}

1. Munkholm P, Langholz E, Davidsen $\mathrm{M}$, et al. Frequency of glucocorticoid resistance and dependency in Crohn's disease. Gut 1994;35:360-2.

2. Munkholm P, Langholz E, Davidsen $\mathrm{M}$, et al. Disease activity courses in a regional cohort of Crohn's disease patients. Scand J Gastroenterol 1995;30:699-706.

3. Faubion WA Jr, Loftus EV Jr, Harmsen WS, et al. The natural history of corticosteroid therapy for inflammatory bowel disease: a population-based study. Gastroenterology 2001;121:255-60.

4. Lapidus A, Bernell O, Hellers G, et al. Clinical course of colorectal Crohn's disease: a 35-year follow-up study of 507 patients. Gastroenterology 1998;114:1151-60.

5. Lofberg R, Rutgeerts $\mathrm{P}$, Malchow $\mathrm{H}$, et al. Budesonide prolongs time to relapse in ileal and ileocaecal Crohn's disease. A placebo controlled one year study. Gut 1996;39:82-6.

6. Candy $\mathbf{S}$, Wright J, Gerber $\mathrm{M}$, et al. A controlled double blind study of azathioprine in the management of Crohn's disease. Gut 1995;37:674-8.

7. Bouhnik Y, Lemann M, Mary JY, et al. Long-term follow-up of patients with Crohn's disease treated with azathioprine or 6-mercaptopurine. Lancet 1996;347:215-9. 
8. Present DH, Korelitz BI, Wisch N, et al. Treatment of Crohn's disease with 6mercaptopurine. A long-term, randomized, double-blind study. N Engl J Med 1980;302:981-7.

9. Feagan BG, Rochon J, Fedorak RN, et al. Methotrexate for the treatment of Crohn's disease. The North American Crohn's Study Group Investigators. N Engl J Med 1995;332:292-7.

10. Feagan BG, Fedorak RN, Irvine EJ, et al. A comparison of methotrexate with placebo for the maintenance of remission in Crohn's disease. North American Crohn's Study Group Investigators. N Engl J Med 2000;342:1627-32.

11. Targan SR, Hanauer SB, Van Deventer SJ, et al. A short-term study of chimeric monoclonal antibody CA2 to tumor necrosis factor alpha for Crohn's disease. Crohn's Disease cA2 Study Group. N Engl J Med 1997;337:1029-35.

12. Hanauer SB, Feagan BG, Lichtenstein GR, et al. Maintenance infliximab for Crohn's disease: the ACCENT I randomised trial. Lancet 2002;359:1541-9.

13. Rutgeerts P, Feagan BG, Lichtenstein GR, et al. Comparison of scheduled and episodic treatment strategies of infliximab in Crohn's disease. Gastroenterology 2004;126:402-13.

14. Present DH, Rutgeerts $P$, Targan S, et al. Infliximab for the treatment of fistulas in patients with Crohn's disease. N Engl J Med 1999;340:1398-405.
15. Sands BE, Anderson FH, Bernstein CN, et al. Infliximab maintenance therapy for fistulizing Crohn's disease. N Engl J Med 2004;350:876-85.

16. Rutgeerts $\mathbf{P}$, Diamond $\mathrm{RH}$, Bala $\mathrm{M}$, et al. Scheduled maintenance treatment with infliximab is superior to episodic treatment for the healing of mucosal ulceration associated with Crohn's disease. Gastrointest Endosc 2006;63:433-47.

17. de Ridder L, Rings EH, Damen GM, et al. Infliximab dependency in pediatric Crohn's disease: long-term follow-up of an unselected cohort. Inflamm Bowel Dis 2008;14:353-8.

18. Poupardin C, Lemann M, Gendre JP, et al. Efficacy of infliximab in Crohn's disease. Results of a retrospective multicenter study with a 15-month follow-up. Gastroenterol Clin Biol 2006;30:247-52.

19. Rudolph SJ, Weinberg DI, McCabe RP. Long-term durability of Crohn's disease treatment with infliximab. Dig Dis Sci 2008;53:1033-41.

20. Pearce CB, Lawrance IC. Careful patient selection may improve response rates to infliximab in inflammatory bowel disease. J Gastroenterol Hepatol 2007:22:1671-7.

21. Fidder $\mathbf{H}$, Schnitzler $F$, Ferrante $\mathbf{M}$, et al. Long-term safety of infliximab in the treatment of inflammatory bowel disease: a single center cohort study. J Crohn's Colitis Supp/ 2007:1:3

\section{Editor's quiz: GI snapshot}

\section{Does old age cause abdominal pain?}

\section{CLINICAL PRESENTATION}

An 87-year-old woman presented with abdominal pain and weight loss of $12 \mathrm{~kg}$. She had been in an excellent state of health until 6 months previously and had not been taking any regular medication. Her family history was unremarkable.

The pain occured in all four abdominal quadrants, was colicky and most severe postprandially. She reported occasional nausea without vomiting. There was a tendency for loose stools.

On examination she was apyrexial and had a body mass index of $16 \mathrm{~kg} / \mathrm{m}^{2}$. Her abdomen was slightly tender on palpation. Cardiovascular and lung findings were normal.

Abdominal ultrasonography, oesophagogastroduodenoscopy, colonoscopy, small bowel imaging, and pancreatic computed tomography (CT) yielded normal results.

Extensive laboratory work-up including urinary porphyrins and C1 esterase inhibitor activity was negative. Adrenal insufficiency was ruled out by an adrenocorticotrophic hormone (ACTH) stimulation test.

Finally, an abdominal CT scan suggested a possible cause of her abdominal pain (fig 1).

\section{QUESTION}

What is the diagnosis?

See page 536 for answer
Robin Spiller, editor

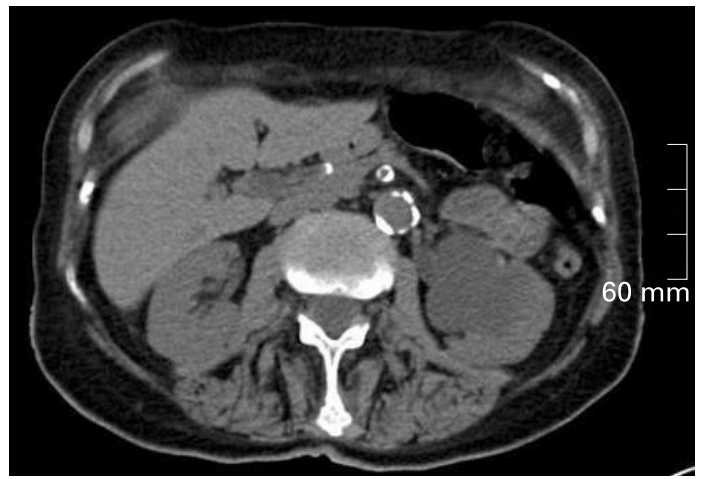

Figure 1 Abdominal CT.

This case is submitted by:

\section{Schiller, M Gschwendtner, R Schöfl}

Elisabethinen Hospital, Linz, Austria

Correspondence to: Dr D Schiller, Elisabethinen Hospital Linz, Fadingerstr. 1, A-4010 Linz, Austria; dietmar.schiller@elisabethinen.or.at

Competing interests: None.

Patient consent: Obtained.

Gut 2009;58:500. doi:10.1136/gut.2008.166975 